\title{
Síndromes de dispersão de diásporos das espécies de trechos de vegetação ciliar do rio das Pacas, Querência - MT
}

\author{
Daniel STEFANELLO ${ }^{1}$, Natália Macedo IVANAUSKAS², Sebatião Venâncio MARTINS³, Elias SILVA, \\ Sustanis Horn KUNZ ${ }^{5}$
}

\section{RESUMO}

As adaptaçóes das plantas, conforme seus agentes dispersores, correlacionam-se com as características morfológicas de cada espécie e família e com a região em que esta predomina, sendo que as sementes evoluíram de acordo com os dispersores. A dispersão de sementes mais freqüente em florestas tropicais é a zoocoria, daí a importância na conservação de corredores ecológicos, que possibilitam a disseminaçáo de espécies de um fragmento para outro. Diante do fato, este trabalho teve por objetivo listar as principais síndromes de dispersão de sementes de espécies ocorrentes em trechos de Floresta Estacional Perenifólia Ribeirinha do rio das Pacas, Querência - MT, a fim de identificar o principal tipo de dispersão. As informações sobre as síndromes de dispersão foram coletadas em campo, juntamente com a amostragem da vegetação, a qual foi dividida em estratos de acordo com a altura dos indivíduos amostrados. Foram identificadas 69 espécies, pertencentes a 51 gêneros e 31 famílias botânicas. As famílias com maior riqueza foram Annonaceae e Fabaceae, a qual apresentou a maior diversificação de síndromes. A zoocoria foi a síndrome de maior expressividade, com $86 \%$ do total de espécies, seguida da anemocoria (10\%), autocoria (3\%) e barocoria (1\%). O estrato inferior (sub-bosque) e intermediário (dossel) possui maior porcentagem de espécies zoocóricas do que no estrato superior (emergente), onde a anemocoria foi mais importante. Estes resultados evidenciam a forte relação entre a vegetação e a fauna na manutenção das populaçóes de plantas no ambiente ciliar, bem como a importância de se manter corredores ecológicos entre os fragmentos florestais.

PALAVRAS-CHAVES: Floresta ciliar, semente, zoocoria, anemocoria

\section{Syndromes of diaspores dispersal of species of riparian vegetation of the river from Pacas, Querência - MT}

\section{ABSTRACT}

The adaptations of plants, as its agents dispersers correlated with the morphological characteristics of each specie and family, and with the region in which it dominates, and the seeds progressed according to the dispersors. The zoochory is the most frequent seed dispersal in the tropical forest, hence the importance in the conservation of ecological corridors, which allowed the spread of species of a fragment to another. Given the fact, this study aimed to trace the major processes of the seed dispersal of species occurring in the Riparian Evergreen Seasonal Forest areas of the Pacas river, Querência - MT, to identify the main dispersal type. The informations on dispersal syndromes were collected in the field during the sampling the vegetation, wich was divided in strata, according to the eight of the individuals sampled. We identified 69 species, belonged 51 genera and 31 botanic families. The families with the greatest richness were Annonaceae and Fabaceae, which showed the greatest variety syndromes. The zoochory were syndrome the more important, with $86 \%$ the total species, followed by anemochory (10\%), autochory (3\%) and barochory (1\%). The lower (understory) and intermediate (canopy) strata have percentage zoochoric species higher than upper stratum (emergente), where the anemochory was the more important. These results demonstrate the strong relationship between the vegetation and animals in the maintenance of plant populations in the riparian environment and the importance of maintaining ecological corridors between forest fragments.

KEYWORDS: Riparian forest, seed, zoochory, anemochory.

\footnotetext{
1 Universidade Federal de Viçosa. E-mail: stefanello.daniel@gmail.com

2 Instituto Florestal do Estado de São Paulo.E-mail: nivanaus@yahoo.com.br

3 Universidade Federal de Viçosa. E-mail: venâncio@ufv.br

${ }^{4}$ Universidade Federal de Viçosa. E-mail: eshamir@ufv.br

${ }^{5}$ Universidade Federal de Viçosa. E-mail: sustanishk@yahoo.com.br
} 


\section{INTRODUÇÃO}

A reprodução de plantas tropicais depende, essencialmente, da interação com animais polinizadores e/ou dispersores de sementes, uma vez que as angiospermas necessitam dos animais para o transporte de pólen ou de suas sementes. Por outro lado, as plantas oferecem recursos alimentares para estes animais: pólen, néctar, óleo, frutos e sementes com polpas suculentas e nutritivas (Morellato e Leitáo-Filho, 1992). As florestas tropicais possuem alta porcentagem de espécies arbóreo-arbustivas (50 a 90\%) que apresentam frutos carnosos, sejam drupas, bagas ou formas ariladas (Frankie et al., 1974; Fleming, 1979).

Assim, o processo de dispersão de sementes é crucial para a reprodução das plantas, pois a semente deve chegar a um local propício para germinar, suficientemente longe da planta-máe, a fim de escapar da competiçáo e também de predadores de sementes e plântulas que ficam nas proximidades da plantamãe (Janzen, 1970; Howe, 1993) o que, por sua vez, acaba influenciando a distribuiçáo espacial dessas plantas (Hubbell, 1979; Howe e Smallwood, 1982; Howe, 1990).

O sucesso de uma espécie em um determinado ambiente depende, em parte, da energia utilizada na sua biologia reprodutiva, ou seja, sucesso na polinização, produçáo de sementes, dispersão e estabelecimento das plântulas (Baker, 1972). Os mecanismos envolvidos na dispersão de sementes de uma planta são adaptações que visam favorecer o seu estabelecimento, sobrevivência e perpetuação (Barradas, 1971; Handro, 1969; Labouriau, 1973), e geralmente dependem do porte do indivíduo e da morfologia de seus frutos e sementes.

O manejo e a recuperação das florestas alteradas dependem da eficiência dos processos de dispersão dos propágulos e do estabelecimento das espécies de diferentes estágios sucessionais, sendo importante na manutenção da regeneração natural durante a dinâmica de sucessão da floresta (Castillo, 1986).

Esta dependência estreita entre planta e dispersor é crucial para a perpetuaçáo das espécies vegetais, uma vez que a remoçáo de um ou outro pode afetar de modo irreversível o equilíbrio das populaçóes (Terborgh, 1986). A interferência em ecossistemas como a remoçáo local da fauna associada, especialmente por pressão de caça, pode levar à extinção ecológica e biológica de espécies vegetais que dependem dos animais para se dispersarem ou mesmo para controlar suas populaçóes (Dirzo e Domingues, 1986; Terborgh, 1986). Além disso, a efetividade da dispersão de sementes está associada ao nível de conservação dos ecossistemas, cuja característica já não é mais observada ao longo do rio das Pacas em uma vegetação ainda pouco estudada.

A bacia hidrográfica do Pacas está sob forte pressão antrópica devido a expansão das fronteiras agrícolas e pecuárias na região nordeste do Estado de Mato Grosso, restando apenas fragmentos florestais mesmo em áreas ciliares, consideradas de preservação permanente. Estas modificaçôes no ambiente promovem náo apenas alteraçôes nos ciclos reprodutivos das plantas, mas afetam também o crescimento e a reprodução dos animais que dependem direta e indiretamente dos recursos vegetais (Howe, 1993). Dada a importância de conhecer as síndromes de dispersáo de sementes, principalmente para programas de restauração florestal, objetivou-se listar os tipos de dispersáo das espécies em trechos de Floresta Estacional Perenifólia Ribeirinha do rio das Pacas em Querência - MT, a fim de identificar o principal tipo de dispersão de sementes nesta unidade vegetacional.

\section{MATERIAL E MÉTODOS}

O estudo foi realizado no Município de Querência (Figura 1), localizado no Vale do Araguaia na posição nordeste do Estado de Mato Grosso, entre os meridianos $52^{\circ} 05^{\prime} 0^{\prime \prime}$ e $13^{\circ}$ $08^{\prime} 5^{\prime \prime} \mathrm{W}$ e os paralelos $11^{\circ} 10^{\prime} 5^{\prime \prime}$ e $13^{\circ} 08^{\prime} 5^{\prime \prime} \mathrm{S}$, a uma altitude de 360m (Corrêa, 1999). O clima da região é classificado como Tropical de Savana (Aw) segundo classificação de Köppen (1948), havendo duas estaçóes bem definidas: a chuvosa, que ocorre no período de outubro a abril; e a seca, que corresponde aos meses de maio a setembro (SEPLAN, 1999). O estudo foi conduzido em um ambiente de Floresta Estacional Perenifólia Ribeirinha, ou seja, área sujeita a inundaçâo no período chuvoso, com presença de lençol freático muito superficial e solos mal drenados.

A coleta dos dados foi realizada em três trechos ao longo do rio das Pacas nos meses de abril, setembro e outubro de 2008. A escolha destes meses foi em função da logística de campo, uma vez que os trechos estudados situam-se em locais distantes do município de Querência. Em cada área foram amostradas 14 parcelas medindo $10 \mathrm{~m}$ de largura por $25 \mathrm{~m}$ de comprimento, totalizando 42 parcelas $\left(10.500 \mathrm{~m}^{2}\right)$. As parcelas foram locadas a partir de $1,0 \mathrm{~m}$ da margem do rio. Foram amostrados todos os indivíduos com circunferência a altura do peito (CAP) $\geq 5$ $\mathrm{cm}$, logo após sendo anotado a altura e realizada a identificaçáo ainda em campo e/ou, quando necessário, realizada a coleta botânica para identificação junto ao herbário.

Para a caracterização da síndrome de dispersão dos diásporos das espécies coletadas, foram utilizados os critérios e categorias propostas por Pijl (1982), reunidas em três grupos básicos: 1) espécies anemocóricas - apresentam mecanismos que facilitam a dispersão pelo vento; 2) zoocóricas - aquelas que possuem características relacionadas à dispersão por animais; e 3) autocóricas - as espécies que dispersam os diásporos por gravidade ou apresentam mecanismos de auto-dispersão, como a deiscência explosiva. A fim de auxiliar a caracterização das síndromes, foi feita uma classificação morfológica dos frutos de acordo com Barroso et al. (1999), para as espécies que apresentaram frutos durante a época de coleta de dados. 


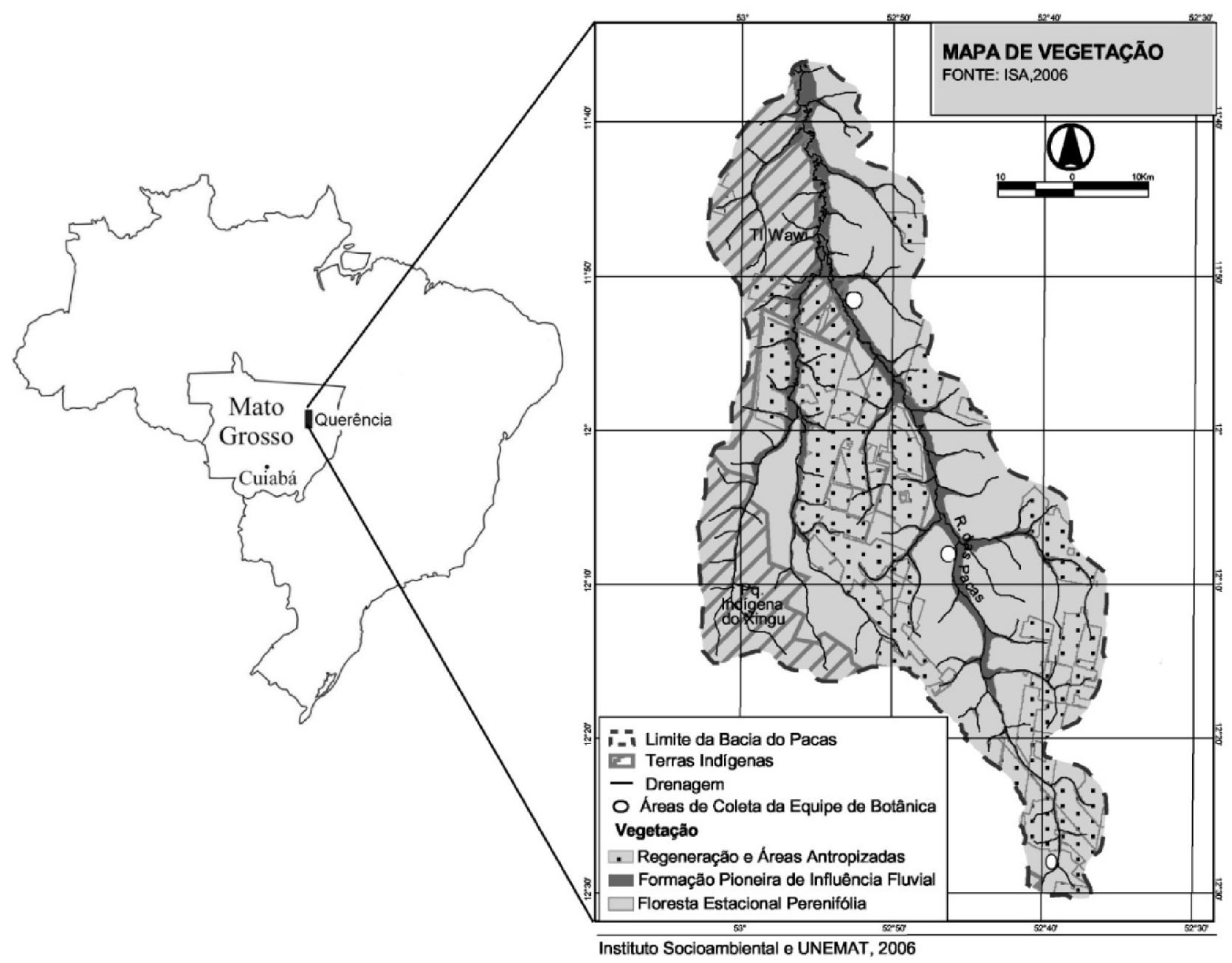

Figura 1 - Localização do município de Querência, com destaque para a bacia do rio das Pacas, MT.

As informaçôes sobre tipo de fruto e síndrome de dispersão foram registradas em campo e através da análise do material coletado durante o estudo. Para as espécies que não frutificaram, foram realizadas consultas à literatura científica e ao material coletado em áreas próximas por outros autores, cujos exemplares estáo depositados no herbário da Coleção Zoobotânica James Alexander Ratter do Campus Universitário de Nova Xavantina/UNEMAT. As espécies que ainda permaneceram sem identificação foram enviadas amostras para especialistas. Após a identificação, o material botânico foi incorporado no herbário da Coleção Zoobotânica James Alexander Ratter do Campus Universitário de Nova Xavantina/UNEMAT.

Para verificar a classificaçáo das espécies dentro dos diferentes estratos da vegetaçáo estudada, considerou-se a altura como critério de inclusão: estrato superior $\geq 20 \mathrm{~m}$ de altura; intermediário $\geq 10,1-19,9 \mathrm{~m}$ e inferior até $10 \mathrm{~m}$ de altura conforme proposto por Ivanauskas et al. (2004).
Cabe destacar que esta classificaçấo considerou apenas os indivíduos adultos de cada espécie e, portanto, que já estão produzindo frutos, e que os mesmos ocuparam os estratos inferiores nas fases de plântula e juvenil.

\section{RESULTADOS E DISCUSSÃO}

Foram registradas 69 espécies, pertecentes a 51 gêneros e 31 famílias botânicas. As famílias com maior riqueza foram Annonaceae e Fabaceae, com nove espécies cada, seguido por Melastomataceae (6) e Burseraceae (5). Entre as famílias amostradas neste estudo, Fabaceae foi a que apresentou a maior diversificaçâo entre as síndromes (Tabela 1).

As florestas tropicais têm como característica apresentar altas proporçôes de espécies vegetais cuja dispersão é feita por animais (zoocoria), com cerca de 70 a $90 \%$ das espécies (Howe e Smallwood, 1982; Stiles, 1989, Morellato e Leitão- 
Tabela 1 - Espécies arbóreas amostradas na Floresta Estacional Perenifólia Ribeirinha do rio das Pacas, Querência-MT, com respectivos tipos de frutos e síndromes de dispersão. Es - Estrato; Int. - Intermediário; Inf. - Inferior; Sup. - Superior

\begin{tabular}{|c|c|c|c|c|}
\hline Es. & Família / Espécie & Nome Vulgar & Tipo de Fruto & Dispersão \\
\hline & ANACARDIACEAE & & & \\
\hline \multirow[t]{2}{*}{ Int. } & Tapirira guianensis Aubl. & Tatapiririca & Drupa & Zoocoria \\
\hline & ANNONACEAE & & & \\
\hline Inf. & Annona amazonica R.E. Fr. & Embira & Múltiplo estrobiliforme & Zoocoria \\
\hline Inf. & Duguetia marcgraviana Mart. & Pindaíba & Sincarpo & Zoocoria \\
\hline Inf. & Ephedranthus amazonicus R.E. Fr. & ----- & Drupa & Zoocoria \\
\hline Int. & Guatteria schomburgkiana Mart. & Cabo-de-rodo & Múltiplo & Zoocoria \\
\hline Int. & Guatteriopsis blepharophylla(Mart.) R.E. Fr. & Envira & Múltiplo livre & Zoocoria \\
\hline Int. & Xylopia amazonica R.E.Fr. & Pindaúva & Apocarpo & Zoocoria \\
\hline Int. & Xylopia crinita R.E.Fr. & Casqueiro & Apocarpo & Zoocoria \\
\hline Int. & Xylopia nitida Dunal & Pindaíba-preta & Apocarpo & Zoocoria \\
\hline \multirow[t]{2}{*}{ Int. } & Xylopia sericea A. St.-Hil & Pindaíba-vermelha & Apocarpo & Zoocoria \\
\hline & APOCYNACEAE & & & \\
\hline \multirow[t]{2}{*}{ Sup. } & Aspidosperma discolor A. DC. & Guarantã & Múltiplo & Anemocoria \\
\hline & ARALIACEAE & & & \\
\hline \multirow[t]{2}{*}{ Sup. } & $\begin{array}{l}\text { Schefflera morototoni (Aubl.) Maguire Steyerm. } \\
\text { \& Frodin }\end{array}$ & Mandiocão & Nuculânio & Zoocoria \\
\hline & ARECACEAE & & & \\
\hline \multirow[t]{2}{*}{ Inf. } & Euterpe edulis Mart. & Palmito & Drupa & Zoocoria \\
\hline & BIGNONIACEAE & & & \\
\hline Sup. & Jacaranda copaia (Auubl.) D. Don & Jacarandá & Múltiplo & Anemocoria \\
\hline \multirow[t]{2}{*}{ Sup. } & Tabebuia impetiginosa (Mart. ex DC.) Standl. & Ipê-roxo & Cápsula loculicida & Anemocoria \\
\hline & BOMBACACEAE & & & \\
\hline \multirow[t]{2}{*}{ Sup. } & Eriotheca candolleana (K. Schum.) A. Robyns & Mamorama & Cápsula loculicida & Anemocoria \\
\hline & BURSERACEAE & & & \\
\hline Inf. & Protium guianense (Aubl.) Marchand & Breu & Filotrimídio & Zoocoria \\
\hline Inf. & Protium pilosissimum Engl. & Breu-peludo & Filotrimídio & Zoocoria \\
\hline Inf. & Protium spruceanum (Benth.) Engl. & Breu & Filotrimídio & Zoocoria \\
\hline Sup. & Trattinnickia burseralifolia Mart. & Amescla & Drupa & Zoocoria \\
\hline \multirow[t]{2}{*}{ Sup. } & Trattinnickia glaziovii Swart. & Mangue & Drupa & Zoocoria \\
\hline & CECROPIACEAE & & & \\
\hline \multirow[t]{2}{*}{ Int. } & Cecropia glaziovi Snethl. & Embaúba & Infrutescência & Zoocoria \\
\hline & CHRYSOBALANACEAE & & & \\
\hline Inf. & Hirtella gracilipes (Hook. f.) Prance & Cariperana & Drupa & Zoocoria \\
\hline Inf. & Hirtella racemosa & Cariperana & Drupa & Zoocoria \\
\hline \multirow[t]{2}{*}{ Int. } & Licania blackii Lam. & Caripé & Drupa & Zoocoria \\
\hline & CONNARACEAE & & & \\
\hline \multirow[t]{2}{*}{ Int. } & Connarus perrottetii Prance & Conaro & Folículo & Zoocoria \\
\hline & ELAEOCARPACEAE & & & \\
\hline \multirow[t]{2}{*}{ Int. } & Sloanea eichleri K. Schum. & Urucurana & Cápsula Loculicida & Zoocoria \\
\hline & EUPHORBIACEAE & & & \\
\hline Int. & Chaetocarpus echinocarpus (Baill.) Ducke & Vermelhinho & Cápsula Septicida & Zoocoria \\
\hline Int. & Maprounea guianensis Aubl. & Marmeleiro & Drupa & Zoocoria \\
\hline Inf. & Pera coccinea (Benth.) Müll. Arg. & Pêra & Cápsula Loculicida & Zoocoria \\
\hline \multirow[t]{2}{*}{ Sup. } & Abarema piresii Barneby \& J.W. Grimes & Ingarana & Legume & Zoocoria \\
\hline & FABACEAE & & & \\
\hline Sup. & Acosmium dasycarpum (Vogel) Yakovlev & Amendoim-falso & Legume Samârideo & Anemocoria \\
\hline Inf. & Bauhinia sp. & Mororó & Legume & Autocoria \\
\hline Sup. & Diplotropis purpurea (Rich.) Amshoff & Sucupira-preta & Legume Samarídeo & Autocoria \\
\hline
\end{tabular}




\begin{tabular}{|c|c|c|c|c|}
\hline Es. & Família / Espécie & Nome Vulgar & Tipo de Fruto & Dispersão \\
\hline Sup. & Enterolobium schomburgkii (Benth.) Benth. & Timbori & Legume & Barocoria \\
\hline Int. & Inga heterophylla Willd. & Ingá-pretinho & Legume Nuculânio & Zoocoria \\
\hline Int. & Inga thibaudiana D.C. & Ingazeiro & Legume Nuculânio & Zoocoria \\
\hline Sup. & Sclerolobium aureum (Tul.) Baill & Carvoeiro & Criptossâmara & Anemocoria \\
\hline \multirow[t]{2}{*}{ Sup. } & Zigia caractae (Kinth) L. Rico & Ingá-chato & Legume & Zoocoria \\
\hline & HIPPOCRATEACEAE & & & \\
\hline \multirow[t]{2}{*}{ Inf. } & Cheiloclinium cognatum(Miers) A.C. Sm. & Uxui-amarelo & Drupa & Zoocoria \\
\hline & HUMIRIACEAE & & & \\
\hline \multirow[t]{2}{*}{ Int. } & Sacoglottis guianensis Benth. & Axuá & Drupóide & Zoocoria \\
\hline & LAURACEAE & & & \\
\hline Int. & Ocotea caudata (Nees) Mez & Canela & Bacáceo & Zoocoria \\
\hline Int. & Ocotea guianensis Aubl. & Louro-prata & Bacáceo & Zoocoria \\
\hline \multirow[t]{2}{*}{ Int. } & Ocotea leucoxylon (Sw.) Laness. & Louro & Bacáceo & Zoocoria \\
\hline & MELASTOMATACEAE & & & \\
\hline Inf. & Miconia acutifolia Ule & Sapateira & Bacídio & Zoocoria \\
\hline Int. & Miconia dispar Benth. & Maramara & Bacídio & Zoocoria \\
\hline Int. & Miconia gratissima Benth. ex Triana & Maramara-branco & Bacídio & Zoocoria \\
\hline Int. & Miconia punctata (Desr.) D. Don ex DC & Tinteiro-branco & Bacídio & Zoocoria \\
\hline Int. & Miconia pyrifolia Naudin & Tinteiro-vermelho & Bacídio & Zoocoria \\
\hline \multirow[t]{2}{*}{ Int. } & Mouriri apiranga Spruce ex Triana & Murici & Bacídio & Zoocoria \\
\hline & MELIACEAE & & & \\
\hline \multirow[t]{2}{*}{ Int. } & Trichilia micrantha Benth. & Cedrinho & Cápsula Loculicida & Zoocoria \\
\hline & MENISPERMACEAE & & & \\
\hline \multirow[t]{2}{*}{ Inf. } & Abuta grandifolia (Mart.) Sandwith & ---- & Drupa & Zoocoria \\
\hline & MONIMIACEAE & & & \\
\hline \multirow[t]{2}{*}{ Inf. } & Siparuna amazonica Mart. ex A. DC. & Maruba & Múltiplo Cupuliforme & Zoocoria \\
\hline & MORACEAE & & & \\
\hline Int. & Brosimum lactescens (S. Moore) C.C. Berg & Leitero & Múltiplo Monocárpico & Zoocoria \\
\hline \multirow[t]{2}{*}{ Int. } & Pseudolmedia murure Standl. & Café-com-Leite & Núcula & Zoocoria \\
\hline & MYRISTICACEAE & & & \\
\hline \multirow[t]{2}{*}{ Int. } & Virola sebifera Aubl. & Ucuúba-de-sangue & Folículo & Zoocoria \\
\hline & MYRTACEAE & & & \\
\hline Int. & Myrcia amazonica DC. & Araçá & Bacáceo & Zoocoria \\
\hline Int. & Myrcia sp. & ---- & Bacáceo & Zoocoria \\
\hline \multirow[t]{2}{*}{ Int. } & Myrcia multiflora (Lam.) DC. & Araçá-peua & Bacáceo & Zoocoria \\
\hline & OCHNACEAE & & & \\
\hline \multirow[t]{2}{*}{ Int. } & Ouratea discophora Ducke & Cumatê & Múltiplo Livre & Zoocoria \\
\hline & QUIINACEAE & & & \\
\hline \multirow[t]{2}{*}{ Int. } & Ouratea discophora Ducke & Cumatê & Múltiplo Livre & Zoocoria \\
\hline & RUBIACEAE & & & \\
\hline \multirow[t]{2}{*}{ Inf. } & Quiina amazonica A.C. Sm. & Quina & Drupa & Zoocoria \\
\hline & RUBIACEAE & & & \\
\hline \multirow[t]{2}{*}{ Inf. } & Amaioua guianensis Aubl. & Canela-de-veado & Anfissarcideo & Zoocoria \\
\hline & SAPINDACEAE & & & \\
\hline Inf. & Matayba arborescens (Aubl.) Radlk & Mata-fome & Cápsula Loculicida & Zoocoria \\
\hline \multirow[t]{2}{*}{ Inf. } & Matayba guianensis Aubl. & Mata-fome & Cápsula Loculicida & Zoocoria \\
\hline & SAPOTACEAE & & & \\
\hline Int. & Pouteria ramiflora (Mart.) Radlk. & Maçarandubinha & Bacóide & Zoocoria \\
\hline Int. & Pouteria torta (Mart.) Radlk. & Maçaranduba & Bacóide & Zoocoria \\
\hline Int. & Micropholis egensis (A. DC.) Pierre & Mangabinha & Drupa & Zoocoria \\
\hline
\end{tabular}




\begin{tabular}{|c|c|c|c|c|}
\hline Es. & Família / Espécie & Nome Vulgar & Tipo de Fruto & Dispersão \\
\hline & TILIACEAE & & & \\
\hline \multirow[t]{2}{*}{ Int. } & Mollia lepidota Spruce ex Benth & ----- & Drupa & Zoocoria \\
\hline & VOCHYSIACEAE & & & \\
\hline Sup. & Vochysia vismiifolia Sprunce ex Warm. & Quarubá-cedro & Cápsula Loculicida & Anemocoria \\
\hline
\end{tabular}

Filho 1992; Tabarelli et al., 1999; Talora e Morellato, 2000), confirmando os resultados do presente estudo (Figura 2).

A alta ocorrência de espécies zoocóricas em áreas de floresta é demonstrada por diversos autores (Howe e Smallwood, 1982; Tabarelli e Peres, 2002; Nunes et al., 2003). Em um remanescente de Floresta Estacional Semidecidual secundária

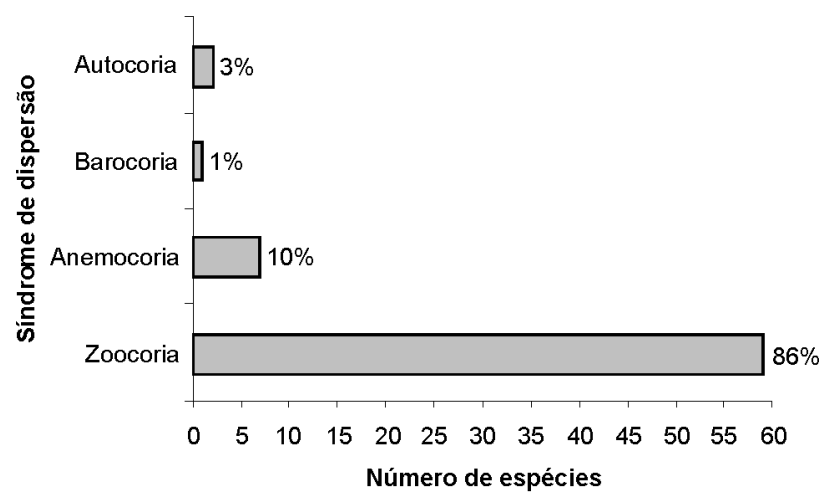

Figura 2 - Síndromes de dispersão nos trechos amostrados de Floresta Estacional Perenifólia Ribeirinha do rio Pacas, Querência - MT.

no Parque Santo Dias - SP, 76\% das espécies apresentaram síndrome de zoocoria, $17 \%$ anemocoria e 6\% autocoria (Garcia e Pirani, 2001). Em 19 Matas de Galeria do Distrito Federal, o número de espécies cuja dispersão é realizada por animais foi superior em todas as áreas comparadas, tendo como resultado geral $72 \%$ de zoocoria, $24 \%$ de anemocoria e apenas uma pequena proporção de espécies autocóricas (3\%) e barocóricas (1\%) (Pinheiro e Ribeiro, 2001).

Resultados semelhantes também foram observados em outras formaçóes vegetais. Em uma área de Floresta Estacional Semidecidual no Paraná, Mikich e Silva (2001) encontraram 58 famílias, das quais 49 são exclusivamente de espécies zoocóricas. Da mesma forma, em uma floresta de brejo na região de Campinas-SP, Spina et al. (2001) encontraram 57\% de espécies zoocóricas, seguidos por $27 \%$ de espécies anemocóricas e $16 \%$ de autocóricas. Já Talora e Morellato (2000) encontraram 89\% de espécies zoocóricas e apenas $10 \%$ de espécies anemocóricas em uma área de Floresta Ombrófila Densa no Parque Estadual da Serra do Mar, em São Paulo.

A alta quantidade de espécies zoocóricas é um indicativo de comunidades vegetais em estádios avançados de sucessão ou que estão em bom estado de conservação (Fenner, 1985), o que garante maior proteçáo e oferta de recursos para a ocorrência de animais. As matas ciliares, por exemplo, favorecem a zoocoria, pois permitem a manutenção de uma fauna característica devido a maior disponibilidade de água e por estarem protegidas do fogo (Ribeiro e Walter, 2001). Isto demonstra a necessidade da preservação das áreas de Floresta Estacional Perenifólia Ribeirinha e de um melhor planejamento para a abertura de áreas onde é permitido, de forma que os fragmentos florestais não sejam pequenos e fiquem conectados, contribuindo para a permanência da fauna local.

A zoocoria parece ser mais significativa no dossel e subbosque da vegetação, pois a maioria das espécies registradas compóe o estrato intermediário $(52,2 \%)$, caracterizando o dossel, seguido pelo estrato inferior (sub-bosque) que contém $27,5 \%$. Já o estrato superior (indivíduos emergentes) é representado por $20,3 \%$ do total de espécies, sendo que neste estrato a anemocoria tem maior expressividade.

A região onde ocorre a Floresta Estacional Perenifólia Ribeirinha é caracterizada por uma estação seca bem definida, mas a vegetação permanece sempre verde, não havendo perda de folhas durante a estiagem (Ivanauskas et al., 2008). Desta forma, fica evidente que apenas as espécies presentes no estrato superior têm maior chance de serem dispersas pelo vento, o que se deve pela maior ação do vento (Oliveira e Moreira, 1992) e ausência de obstáculos (Putz, 1983). De acordo com Howe e Smallwood (1982), a zoocoria e a anemocoria estão relacionadas ao habitat, pois a primeira tem maior importância em ambientes úmidos, enquanto a segunda prevalece em ambientes secos ou que apresentam certa sazonalidade. Neste caso, a anemocoria é facilitada pela caducidade foliar durante a estação seca (Mantovani e Martins, 1988), o que não é característico da vegetação estudada.

A produção de sementes pequenas é mais vantajosa do que frutos grandes e carnosos, pois eleva a quantidade de sementes ingeridas pelos animais (Graham et al., 1995). Além disso, o sucesso de estabelecimento da planta no local de deposição da semente depende de fatores ambientais apropriados para o estabelecimento de um novo indivíduo, fato este que conduz as plantas a produzirem sementes em grande quantidade (Pijl, 1972). De fato, algumas espécies zoocóricas encontradas no presente estudo apresentam alta abundância e freqüência em trechos de interflúvio (Kunz et al., 2008), o que pode estar correlacionado a alta produçáo de 
sementes pequenas e ao ambiente favorável à sua germinação. Dentre elas, pode-se citar Xylopia amazonica, Trattinnickia glaziovii, Schefflera morototoni, Chaetocarpus echinocarpus, Trichilia micrantha, Pseudolmedia murure, Ocotea guianensis e Myrcia amazonica, as quais exibem frutos de cores vistosas com polpas ou arilos carnosos e suculentos, características que indicam uma adaptação para a ornitocoria, um tipo de dispersão zoocórica.

As síndromes de dispersão encontradas no estudo estão relacionadas ao tipo de fruto das espécies da comunidade. Entre as 69 espécies amostradas na floresta ciliar foram encontrados 23 tipos diferentes de frutos. Aqueles com maior ocorrência foram drupas, que representaram $20,3 \%(\mathrm{~N}=14)$, cápsula loculicida com 10,1\% (N=7), bacídeo e bacáceo com $8,7 \%(\mathrm{~N}=6)$, respectivamente, e apocarpo com $5,8 \%(\mathrm{~N}=4)$. Os demais representaram $46,4 \%(\mathrm{~N}=32)$ do total de espécies. Os frutos carnosos, como é o caso da drupa, bacídeo e bacáceo registrados neste estudo, sáo freqüentemente comestíveis e por isso altamente atrativos para os animais, o que favorece a dispersão. O fruto tipo drupa, por exemplo, possui um epicarpo e mesocarpo com consistência carnosa e com um só pirênio (caroço) no seu interior (Barroso et al., 1999).

Frutos secos também podem indicar dispersão zoocórica quando possuem mecanismos especiais, como ganchos ou substâncias aderentes, que se prendem facilmente ao pêlo dos animais para serem transportados (Howe e Smallwood, 1982; Raven et al., 2001). Esta ampla variação morfológica dos frutos na mesma síndrome revela a variedade de estratégias que as plantas possuem para atrair os dispersores, os quais, por sua vez, podem ser beneficiados pela maior disponibilidade de recurso alimentar (Pinheiro e Ribeiro, 2001). Infelizmente, é difícil fazer comparaçóes e apresentar maiores conclusōes a respeito deste assunto, pois não existem muitos estudos sobre as proporçôes dos tipos de frutos em matas ciliares. Além disso, pouco se conhece sobre as espécies que compóem a Floresta Estacional Perenifólia Ribeirinha, o que dificulta a classificação em mais de um tipo de dispersão.

No entanto, esta variedade de frutos, principalmente aqueles zoocóricos, indica a necessidade de preservação da fauna local. Por isso, a conservação de fragmentos com diversos tamanhos, assim como o estabelecimento de corredores para a conexão de paisagens são muito importantes para restabelecer a circulaçáo de animais (Tabarelli e Gascon, 2005), incluindo frugívoros especialistas, que são indispensáveis para a manutenção e futuro das espécies restritas da Floresta Amazônica.

\section{CONCLUSÕES}

A principal síndrome de dispersão de sementes foi a zoocoria, o que mostra uma estreita relação entre a vegetação arbórea e a fauna na manutençâo das populaçôes de plantas no ambiente de vegetação ciliar.

Matas ciliares funcionam como corredores ecológicos, possibilitando o acesso de animais à água, e fornecendo abrigo e alimentos para estes. Assim, o destaque da zoocoria neste estudo vem reforçar a importância do cumprimento da legislação florestal sobre a obrigatoriedade da conservaçáo das florestas ribeirinhas, que particularmente nesta região do Estado de Mato Grosso nem sempre tem sido acatado.

A Floresta Estacional Perenifólia Ribeirinha ainda é muito pouco conhecida quanto a sua ecologia e, por isso, mais estudos ecológicos são necessários para melhor entendimento da dinâmica das espécies florestais. Somente a partir disso é que será possível formular planos de manejo a fim de que as funçóes ecológicas neste ambiente táo importante sejam mantidas, bem como embasar os projetos de restauração florestal nas áreas já degradadas.

\section{AGRADECIMENTOS}

Agradecemos à Agência dos Estados Unidos para Desenvolvimento Internacional (USAID), Consórcio Estradas Verdes e Fundação de Amparo à Pesquisa do Estado de Mato Grosso (FAPEMAT - Processo 08/2004) pelo apoio financeiro para o desenvolvimento do Projeto Gestão Ambiental e Ordenamento Territorial da Bacia do rio SuiáMiçu. Ao Programa Xingu/ISA (Instituto Socioambiental) e Universidade do Estado de Mato Grosso (UNEMAT) pelo apoio técnico-científico e logístico.

\section{BIBLIOGRAFIA CITADA}

Baker, H.G. 1972. Seed weight in relation to environmental conditions in California. Ecology, 53: 998-1010.

Barradas, M.M. 1971. Estrutura do fruto e da semente do pequi Caryocar brasiliense Camb. (Caryocaraceae). Dissertação de Mestrado, Universidade de São Paulo, São Paulo. 61 pp.

Barroso, G.M.; Morin, M.P.; Peixoto A.L.; Ichaso, C.L.F. 1999. Frutos e sementes: morfologia aplicada à sistemática de dicotiledôneas. Editora UFV, Viçosa. 443 pp.

Castillo, C.A.R. 1986. Dispersão anemocórica das sementes de paineira (Chorisia speciosa St. Hil.) na região de Bauru, Estado de São Paulo. Dissertaçáo de Mestrado, Escola Superior de Agricultura Luiz de Queiroz, Piracicaba, São Paulo. 140 pp. 
Corrêa, J.C. 1999. Manejo do solo no município de Querência, MT. Embrapa Cerrados, Planaltina. 56 pp.

Dirzo, R.; Dominguez, C.A. 1986. Seed shadows, seed predation and the advantages of dispersal, p. 237-249. In: Estrada, A.; Fleming, T.H. (Eds.). Frugivores and seed dispersal. W. Junk, Dordrech.

Fenner, F. 1985. Seed ecology. Champman and Hall, London. 151 pp.

Fleming, T.H. 1979. Do tropical frugivores completed for food? Annales Zoologici Fennici, 19: 1157-72.

Frankie, G.W.; Baker, H.G.; Opler, P.A. 1974. Comparative phonological studies of trees in tropical wet and dry forests en the lowands of Costa Rica. Journal of Ecology, 62: 881-919.

Garcia, R.J.F.; Pirani, J.R. 2001. Estudo florístico do componente arbóreo e arbustivo da Mata do Parque Santo Dias, São Paulo, SP, Brasil. Boletim de Botânica da Universidade de São Paulo, 19: 15-42.

Graham, C.H.; Moermond, T.C.; Kristenses, K.A.; Mvukiyumwami, J. 1995. Seed dispersal effectiveness by two bulbuls on Masea lanceolata, an African montane forest tree. Biotropica, 27: 479486.

Handro, W. 1969. Contribuição ao estudo da unidade de dispersão e da plântula de Andira humilis Mart. ex. Benth (Leguminosae - Lotoideae). Anais da Academia Brasileira de Ciências, 41: 286-287.

Howe, H.F. 1990. Seed dispersal by birds and mammals implications for seedling demography, p. 191-218. In: Bawa, K.S.; Hadley, M. (Eds.). Reproductive ecology of tropical forest plants. Man and the biosphere series. Vol. 7. UNESCO \& Parthenon Publishing Group, Paris.

Howe, H.F. 1993. Aspects of variation in a Neotropical seed dispersal system. Vegetatio, 107/108: 149-162.

Howe, H.F.; Smallwood, J. 1982. Ecology of seed dispersal. Annual Review of Ecology and Systematic, 13: 201-228.

Hubell, S.P. 1979. Tree dispersion, abundance, and diversity in a tropical dry forest. Science, 203: 1299-1309.

Ivanauskas, N.M.; Monteiro, R.; Rodrigues, R.R. 2004. Estrutura de um trecho de floresta amazônica na bacia do alto rio Xingu. Acta Amazonica, 34: 275-299.

Ivanauskas, N.M., Monteiro, R.; Rodrigues, R.R. 2008. Classificaçâo fitogeográfica das florestas do Alto Rio Xingu. Acta Amazonica, 38: 387-402.

Janzen, D.H. 1970. Herbivories and the number of tree species in tropical forests. American Naturalist, 104: 501-528.

Köppen, W.P. 1948. Climatologia: com um estudio de los climas de la tierra. Fondo de Cultura Econômica, México. 478 pp.

Kunz, S.H.; Ivanauskas, N.M.; Martins, S.V.; Silva, E.; Stefanello, D. 2008. Aspectos florísticos e fitossociológicos de um trecho de Floresta Estacional Perenifólia na Fazenda Trairão Bacia do rio das Pacas, Querência - MT. Acta Amazonica, 38: 245-254.

Labouriau, M.L.S. 1973. A semente de Magonia pubescens St. Hil. - Morfologia e germinação. Academia Brasileira de Ciências, 45: 501-537.
Mantovani, W.; Martins, F.R. 1988. Variações fenológicas das espécies do cerrado da Revista Biológica de Moji Guaçu, Estado de São Paulo. Revista Brasileira de Botânica, 11: 101-112.

Mikich, S.B.; Silva, S.M. 2001. Composição Florística e Fenologia das Espécies Zoocóricas de Remanescentes de Floresta Estacional Semidecidual no Centro - Oeste do Paraná, Brasil. Acta Botanica Brasilica, 15: 89-113.

Morellato, L.P.C.; Leitão-Filho, H.F. 1992. Padrões de frutificação e dispersão na Serra do Japi, p. 112-141. In: Morellato, L.P.C. (Org.) História Natural da Serra do Japi: ecologia e preservação de uma área florestal no Sudeste do Brasil. Editora da UNICAMP/ FAPESP, Campinas.

Nunes, Y.R.F.; Mendonça, A.V.R.; Machado, E.L.M.; Oliveira-Filho, A.T. 2003. Variaçôes da fisionomia, diversidade e composição de guildas da comunidade arbórea em um fragmento de Floresta Semidecidual em Lavras - MG. Acta Botanica Brasilica, 17: 213-229.

Oliveira, P.E.A.M.; Moreira, A.G. 1992. Anemocoria em espécies de cerrado e mata de galeria de Brasília - DF. Revista Brasileira de Botânica, 15: 163-174.

Pijl, V.D.L. 1982. Principles of dispersal in higher plants. 3 ed. Springer Verlag, New York. 402 pp.

Pinheiro, F.; Ribeiro, J.R. 2001. Síndromes de dispersão de sementes em Matas de Galeria do Distrito Federal, p. 335-361. In: Ribeiro, J.F.; Fonseca, C.E.L.; Sousa-Silva, J.C. (Eds.). Cerrado: caracterizaçâo e recuperaçáo de matas de galeria. Embrapa Cerrados, Planaltina.

Putz, F.E. 1983. Liana biomass and leaf area of a Tierra Firme forest in the Rio Negro basin, Venezuela. Biotropica, 15: 185-189.

Raven, P.H.; Evert, R.F.; Eichhorn, S.E. 2001. Biologia vegetal. 6 ed. Guanabara Koogan, Rio de Janeiro. 906 pp.

Ribeiro, J.F.; Walter, B.T.M. 2001. As matas de galeria no contexto de bioma Cerrado, p. 29-45. In: Ribeiro J.F.; Fonseca, C.E.L. \& Sousa-Silva, J.C. (Eds.). Cerrado: caracterização e recuperação de matas de galeria. Embrapa Cerrados, Planaltina.

SEPLAN/MT. 1999. Dados secundários do DSEE/MT: Zoneamento - Divulga. CD-Rom. Versão 1.01.

Spina, A.P.; Ferreira, W.M.; Leitão-Filho, H.F. 2001. Floração, frutificação e síndrome de dispersão de uma comunidade de Floresta de Brejo na regiáo de Campinas (SP). Acta Botanica Brasilica, 15: 349-368.

Stile, E.W. 1989. Fruits, seeds and dispersal agents. In: Abraham, W.G. Plant - animal interactions. Mc Graw Hill, New York. $351 \mathrm{pp}$.

Tabarelli, M.; Gascon, C. 2005. Lessons from fragmentation research: improving management and policy guidelines for biodiversity conservation. Conservation Biology, 19: 734-739.

Tabarelli, M.; Peres, C.A. 2002. Abiotic and vertebrate seed dispersal in the Brazilian Atlantic forest: implications for forest regeneration. Biological Conservation, 106: 165-176.

Tabarelli, M.; Mantovani, W.; Peres C.A. 1999. Effects of habitat fragmentation on plant guild structure in the montane Atlantic forest of southeastern Brazil. Biological Conservation, 91: 119127. 


\section{ACTA}

Talora, D.C.; Morellato, L.P.C. 2000. Fenologia de espécies arbóreas em floresta de planície litorânea do sudeste do Brasil. Revista Brasileira de Botânica, 23: 13-26.

Terborgh, J. 1986. Community aspects of frugivory in tropical Forest, p. 371-384. In: Estrada, A.; Fleming, T.H. (Eds.). Frugivores and seed dispersal. W. Junk, Dordrech.

Recebido em 01/09/2008

Aceito em 16/09/2009 
